\title{
Estudio de la ladera occidental del Cerro del Chiquihuite, Ciudad de México mediante SIG y GPS
}

\author{
GPS and GIS study of the western slope of the \\ Chiquihuite hill in Mexico City
}

\author{
M. Martínez-Yáñez \\ Instituto de Geofísica, UNAM, México. \\ Schlumberger, Oilfield Services, México. \\ E-mail:mayma@geofisica.unam.mx \\ E. Cabral-Cano \\ Instituto de Geofísica, UNAM, México. \\ E-mail:ecabral@igeofcu.unam.mx \\ F. Correa-Mora \\ Instituto de Geofísica, UNAM, México. \\ E-mail:fcorrea@geology.wisc.eduo \\ O. Díaz-Molina \\ Instituto de Geofísica, UNAM, México. \\ E-mail:oscard@geofisica.unam.mx \\ G. Cifuentes-Nava \\ Instituto de Geofísica, UNAM, México. \\ E-mail:gercifue@geofisica.unam.mx \\ E. Hernández-Quintero \\ Instituto de Geofísica, UNAM, México. \\ E-mail:estebanh@geofisica.unam.mx \\ H. Delgado-Granados \\ Instituto de Geofísica, UNAM, México. \\ E-mail:hugo@geofisica.unam.mx
}

(Recibido: agosto de 2006; aceptado: mayo de 2008)

\section{Resumen}

La explosión demográfica en la Ciudad de México ha obligado a que su población se haya asentado en las laderas con pendientes abruptas, generando un crecimiento anárquico del espacio urbano. La topografía del Cerro del Chiquihuite, es el elemento principal que condiciona el riesgo para los pobladores con la posibilidad de rodamientos de rocas y deslizamientos de laderas. Con el fin de establecer un marco de referencia para detectar movimientos a mediano y largo plazo se desarrolló una red geodésica conformada por 12 sitios y una estación de referencia permanente (UCHI) ubicada en la cabecera Sur del Cerro del Chiquihuite. La red del Cerro del Chiquihuite fue monitoreada durante el periodo comprendido de octubre del $2000 \mathrm{a}$ enero del 2006, mediante campañas realizadas con intervalos variables. Sus resultados fueron obtenidos por medio de procesamiento diferencial con solución de ambigüedades y órbitas precisas, y utilizando la estación UCHI como referencia. La red de estaciones GPS en el Cerro del Chiquihuite no muestra variaciones significativas, por lo que se concluye que en este lapso no existen movimientos sustanciales 
DOI: http://dx.doi.org/10.22201/fi.25940732e.2009.10n2.010

Estudio de la ladera occidental del Cerro del Chiquihuite, Ciudad de México mediante SIG y GPS

del talud. El único sitio que muestra variación significativa en su componente vertical es la estación CH55 que muy probablemente se encuentre afectada por procesos de subsidencia regional.

Descriptores: GPS, riesgogeológico, Chiquihuite, DistritoFederal.

\begin{abstract}
The demographic explosion of the City of Mexicohas forced the anarchical growth of urban development in its mountain slopes. The geologic risk conditions that prevail in such areas are rock falls and down slope creep. The topographic slope analysis shows that these some areas pose a high risk condition for the housing developments located down slope. A geodetic network was thus developed for the establishment of a reference frame to detect medium and long term slope movement. The location of these benchmarks included rock outcrops, structural containment civil structures and street sidewalks. This network was designed to be occupied using GPS fast static methods, with times of occupation no greater to 45 minutes per station. In order to keep short baselines to the reference station and its position errors within low levels we installed a reference GPS site (UCHI) on the Southern part of Cerro del Chiquihuite. The Chiquihuite GPS network was monitored for 5 years. The GPS solutions were obtained by differential techniques with ambiguity solution and precise orbits, and using UCHI stations as a reference. The Chiquihuite GPS network does not show significant variations, except for the vertical component at station CH55. This site is likely to be affected by regional subsidence.
\end{abstract}

Keywords: GPS, geological hazard, Chiquihuite, Distrito Federal.

\section{Introducción}

La Ciudad de México presenta muchos de los riesgos derivados de un desarrollo urbano impetuoso dentro de una situación de fuertes contrastes económicos y poca planeación de su crecimiento (Salazar, 1996). Ejemplo de esto son las áreas de fuerte pendiente que se encuentran en proceso de urbanización. Es frecuente que los daños a casas habitación reflejen la magnitud de los movimientos del terreno y de las áreas donde el problema es crítico, como consecuencia de un crecimiento sin control y en lugares poco apropiados. La explosión demográfica en la ciudad ha obligado a que su población se haya asentado en las laderas con pendientes abruptas, generando un crecimiento anárquico del espacio urbano. Las condiciones de riesgo que prevalecen en las zonas de laderas son la posibilidad de rodamientos de rocas y deslizamientos de laderas. Estas condiciones amenazan constantemente a la población en una situación que se ve agravada en la temporada de lluvias (figura 2a). Un ejemplo claro de este tipo de riesgos se observan en el Cerro del Chiquihuite, ubicado en los límites de la Delegación Gustavo A. Madero (Distrito Federal) y municipio de Tlalnepantla, Estado de México, (figura 1) en donde se han tratado de regularizar varios asentamientos no planificados.
Por lo anterior, se consideró importante monitorear la ladera occidental mediante estudios geodésicos utilizando técnicas de GPS y determinar la posible existencia de deslizamientos en las laderas del Cerro del Chiquihuite. Esto se llevó a cabo mediante la creación de una red geodésica formada por 12 estaciones de control, que fueron monitoreadas en campañas realizadas periódicamente durante 26 meses.

\section{Área de estudio}

El Cerro del Chiquihuite (figura 1), en el límite Norte del Distrito Federal, forma parte de la Sierra de Guadalupe, junto con los Cerros El Tepeyac, Guerrero y Santa Isabel constituida esencialmente de lavas de composición intermedia, aunque la morfología del conjunto de volcanes que constituyen la Sierra de Guadalupe ha sido alterada sustancialmente, quizá con excepción de los volcanes Tenayo y Chiquihuite, que son más jóvenes. En especial, aquellos que constituyen el parteaguas han perdido su morfología original y están disectados por numerosos barrancos en los que se han desarrollado circos de erosión. Es evidente un rápido retroceso actual de las laderas empinadas con la presencia de escarpes (Lugo, 1996). 


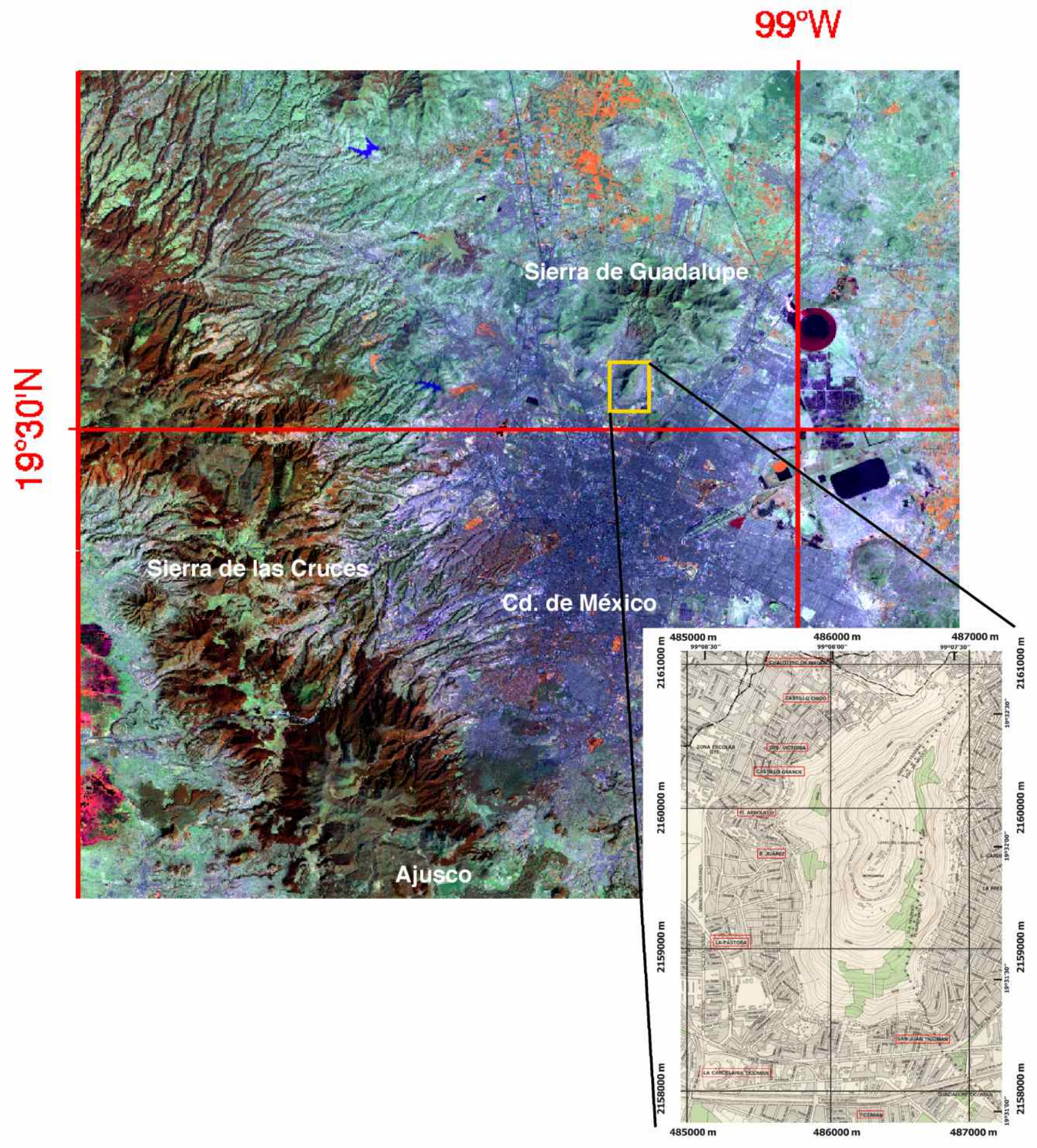

Figura 1. Imagen ASTER que muestra la localización del Cerro del Chiquihuite al Norte de la ciudad de México (recuadro) en la Delegación Gustavo A. Madero y municipio e Tlalnepantla, Distrito Federal y Estado de México 


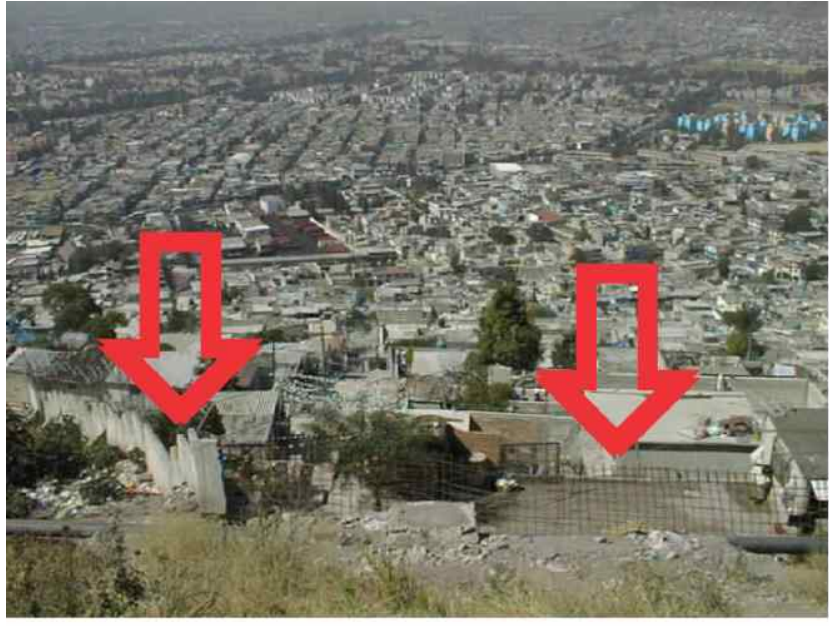

a)

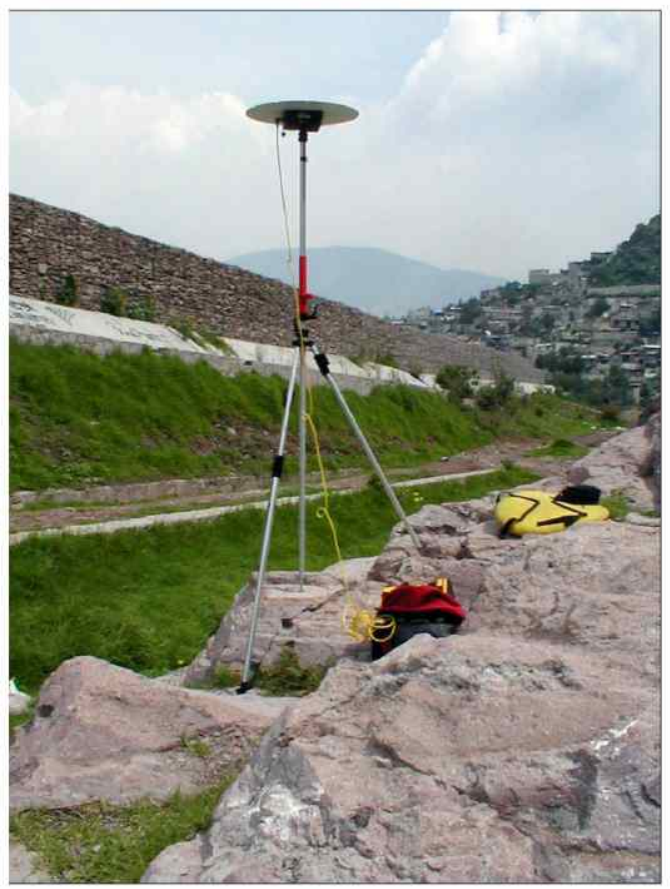

c)

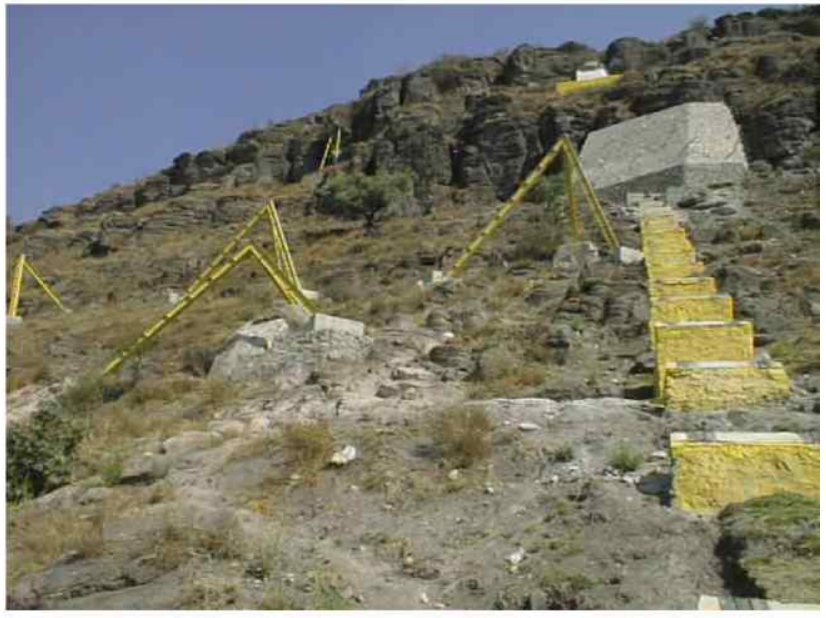

b)

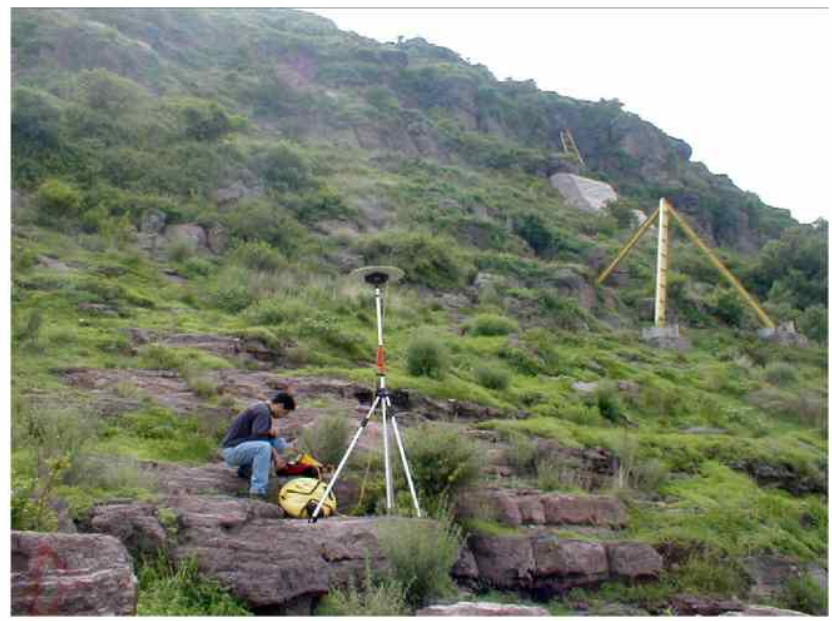

d)

Figura 2. a) Daño en la barda que delimita la reserva ecológica debido a bloques de roca desprendido. b) Elementos de contención en la ladera occidental del Cerro del Chiquihuite

c) yd) Levantamientos de estaciones GPS en el Cerro del Chiquihuite 
DOI: http://dx.doi.org/10.22201/fi.25940732e.2009.10n2.010

M. Martínez-Yáñez, E. Cabral-Cano, F. Correa-Mora, O. Díaz-Molina, G. Cifuentes-Nava, E. Hernández-Quintero, H. Delgado-Granados

La morfología de la Sierra de Guadalupe muestra que la denudación se ha llevado a cabo por erosión remontante. Algunos valles montañosos han pasado a un proceso de erosión lateral y acumulación; el mayor sigue una dirección NE y se sitúa entre los volcanes Tenayo y Chiquihuite, con dimensiones de 4.5 por $1.5 \mathrm{~km}$. Los valles erosivos, de fondo estrecho y perfil en "V", se disponen en forma radial y sus cabeceras se aproximan a la divisoria. La disección de las vertientes montañosas es de 2.5 a $3 \mathrm{~km} / \mathrm{km}^{2}$ (Lugo, 1981) y la profundidad de corte de 100 a $300 \mathrm{~m}$, valores elevados si se considera que el desnivel entre las cimas y la planicie lacustre es de 400-700 m. Los valles montañosos de esta sierra presentan cabeceras amplias de diámetro de 0.5 a $2 \mathrm{~km}$. Predomina el escurrimiento fluvial sobre la infiltración, intenso durante el verano; el promedio anual de precipitaciones pluviales es del orden de 630-800 mm. (Lugo, 1990). La variación natural de la Sierra de Guadalupe se caracteriza por tener usos de tierra predominantemente forestal y de afloramientos diversos con alto índice de deforestación y de asentamientos irregulares que propician la erosión, lo cual pone en peligro el equilibrio ecológico de la zona. Su clima es húmedo y por su altitud que es de $2700 \mathrm{msnm}$. es una zona fría, tiene escurrimientos que desembocan en las barrancas y forman pequeños arroyos en las colonias y los barrios de Cuautepec, que son encausados como drenaje pluvial hasta el vaso regulador del Arbolillo.

\section{Vulnerabilidad a la inestabilidad del talud}

El Cerro del Chiquihuite es un ejemplo de lo inadecuado que puede ser el establecimiento irregular de cientos de familias a lo largo de zonas no aptas para zonas habitacionales. Uno de los hechos que marcaron el inicio de acciones de mitigación de peligro más recientes fue la caída de un bloque de roca en 1998, que rodó hasta la zona urbanizada y afectó varias viviendas en la calle Coatlicue en la colonia La Pastora. Las autoridades delegacionales comenzaron a implementar medidas diversas para evitar que más rocas se desprendieran. En 1999, la Delegación Gustavo A. Madero (DGAM) inició la construcción de un muro de amortiguamiento en el Cerro del Chiquihuite

A fines de junio del 2000, un desprendimiento en la ladera Este del Cerro del Chiquihuite provocó la muerte de una persona a consecuencia de la caída de toneladas de rocas, lodo y escombros de casas afectadas por las intensas lluvias que se registraron en la colonia Lázaro Cárdenas segunda sección. En otra ocasión una fuerte lluvia provocó el desprendimiento de una barda de 40 metros de largo por 15 metros de alto, ubicada en la cara Noroeste del Cerro del Chiquihuite, lo que ocasionó el derrumbe de cuatro casas y afectó una franja de 20 metros de longitud, con un área aproximada de 600 metros cuadrados.

El estado en que se encuentran los habitantes de los alrededores del Cerro del Chiquihuite sigue siendo de alto riesgo. A pesar de las medidas para reforzar la seguridad éstas no necesariamente resultan suficientes, sobre todo porque se sigue extendiendo la mancha urbana. El Cerro del Chiquihuite es una de la zonas en donde el riesgo existente pone en peligro a un gran número de habitantes, tanto en la DGAM como del municipio de Tlalnepantla. La DGAM ocupa el $11^{\circ}$ lugar entre las 16 delegaciones del Distrito Federal en densidad poblacional con 145.1 habitantes/ha en 1995. Sin embargo, dentro del territorio de la delegación se tienen áreas tanto de alta como de baja densidad. En consecuencia, un número significativo de familias se encuentran en riesgo de sufrir eventos como los anteriormente descritos.

\section{Análisis de peligro en el Cerro Chiquihuite}

La topografía del Cerro del Chiquihuite es el elemento principal que condiciona el peligro potencial y el riesgo para los pobladores, como se puede observar en la figura 3a. En épocas de lluvia, las intensas precipitaciones aunadas a las fuertes pendientes, pueden llegar a originar eventos de gran impacto.

Para caracterizar esta situación se determinaron las zonas de escorrentías (figura 3b).

Como se puede observar en la figura $3 \mathrm{c}$, la cual muestra el análisis de pendientes, el crecimiento de la mancha urbana se ha ido extendiendo y en la actualidad muchos pobladores se encuentran en zonas de alto riesgo. La figura $3 d$, muestra el análisis de la distancia existente entre las pendientes de mayor inclinación y los inmuebles más cercanos. Este análisis se obtuvo al crear envolventes a las zonas señaladas de mayor riesgo por pendientes. Las distancias generadas para ejemplificar la proximidad son de $10 \mathrm{~m}$ y $50 \mathrm{~m}$ y muestran viviendas y pendientes críticas. Se puede observar también que la porción del Cerro Chiquihuite que presenta mayor área de fuertes pendientes, es la ladera Norte. Estos resultados indican que en los planes de desarrollo debería evitarse la urbanización de esta zona.

En la figura 3e se sobrepone el análisis de pendientes con la localización de escorrentías que se muestran como polígonos generados a partir de los parteaguas. El análisis de esta figura nos muestra que dichas zonas representan sectores del talud con alto índice de peligro 
para los inmuebles ubicados pendiente abajo de estos sectores. La intersección de la zonas de afectación de las escorrentías con las áreas de gran pendiente delimitan

a)

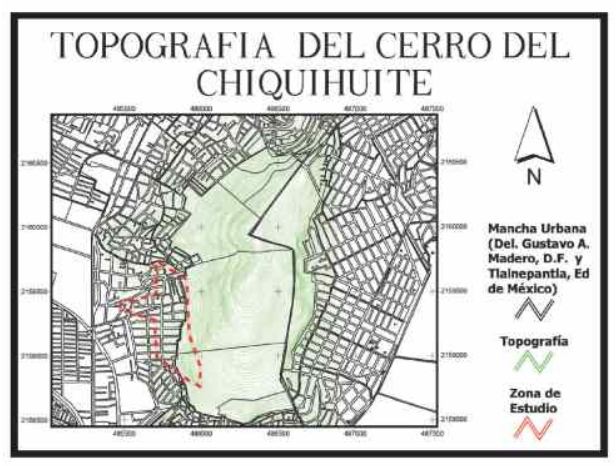

c)

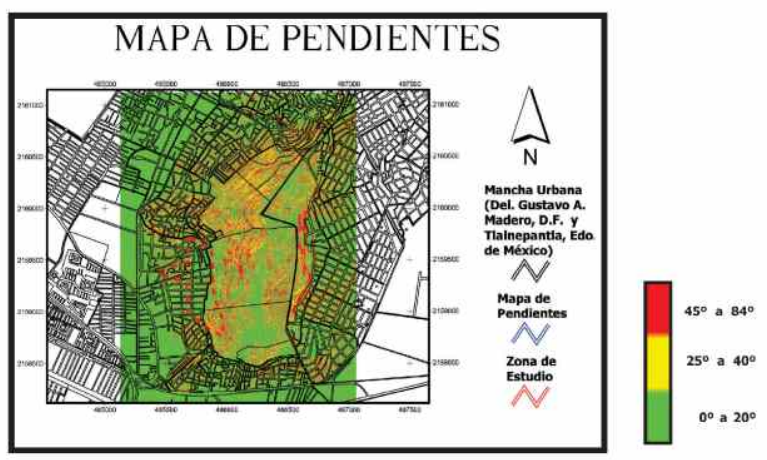

e)

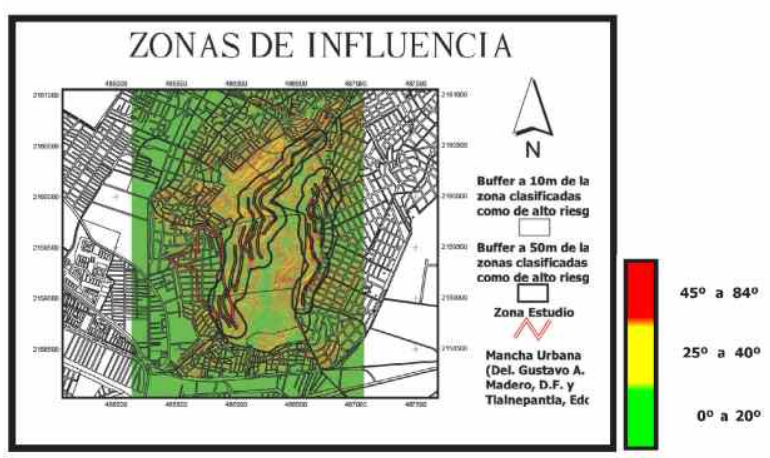

los sectores de mayor peligro para el Cerro del Chiquihuite (figura 3f).

b)

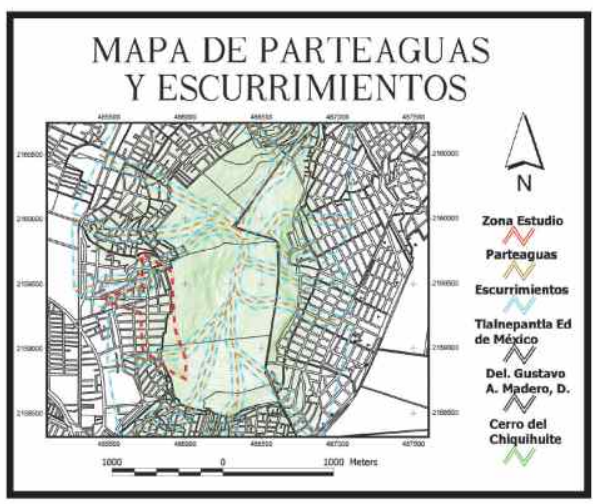

d)

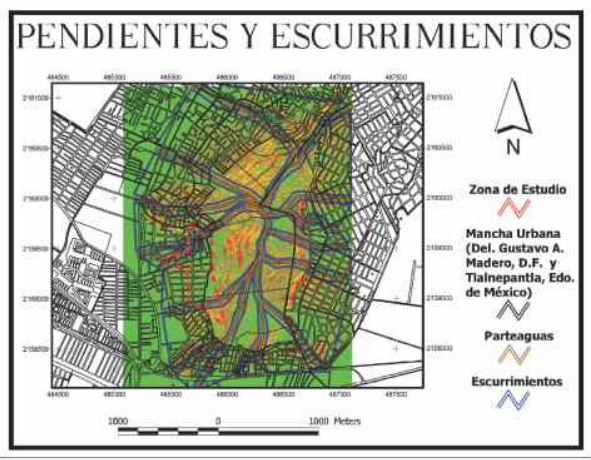

f)

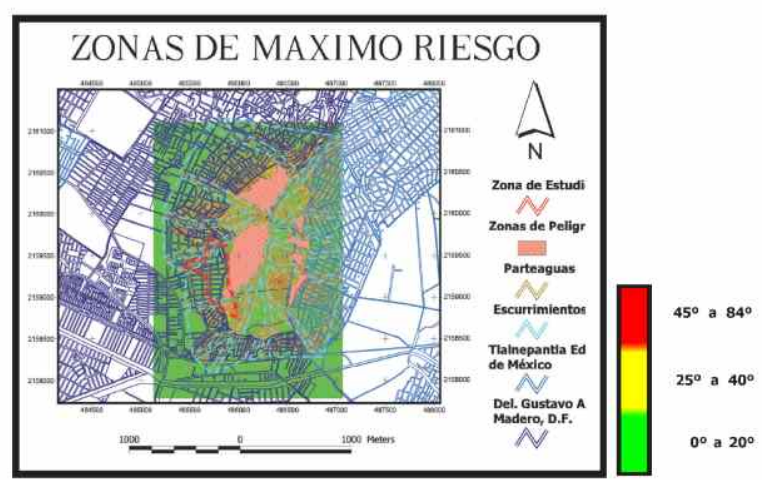

Figura 3. Análisis de pendiente topográfica en el Cerro del Chiquihuite 
DOI: http://dx.doi.org/10.22201/fi.25940732e.2009.10n2.010

M. Martínez-Yáñez, E. Cabral-Cano, F. Correa-Mora, O. Díaz-Molina, G. Cifuentes-Nava, E. Hernández-Quintero, H. Delgado-Granados

\section{Monitoreo de estabilidad del talud}

El peligro de inestabilidad del talud occidental del Cerro del Chiquihuite sobre el cual se ha construido la colonia La Pastora es una situación que resalta en la zonificación de la figura $3 f$. Con el fin de establecer un marco de referencia para poder detectar movimientos a mediano y largo plazo en esta ladera, se procedió a desarrollar una red geodésica, conformada por 12 sitios (figuras 2c, 2d y 4). En estos puntos se colocaron testigos de acero inoxidable que fueron fijados con resina epóxica. Su posición incluyó afloramientos de roca, elementos de contención y guarniciones de banquetas sobre algunas calles. Como esta red fue diseñada para ser ocupada en modo estático rápido, con tiempos de ocupación no mayores a 45 minutos por estación, el uso de una estación de referencia cercana es muy útil para mantener líneas de base cortas $y$, por lo tanto, mantener errores dentro de niveles bajos. Por esta razón se instaló una estación de referencia permanente (UCHI) ubicada en la cabecera Sur del Cerro del Chiquihuite, El posicionamiento de la estación UCHI fue realizado mediante GIPSY por posicionamiento de punto preciso y utilizando orbitas precisas y soluciones de ambigüedades siguiendo el procedimiento descrito por Sella et al. (2002).

La red del Cerro del Chiquihuite fue monitoreada durante un periodo mayor a los 5 años. Sus resultados fueron obtenidos por medio de procesamiento diferencial con solución de ambigüedades y utilizando órbitas precisas utilizando la estación UCHI como referencia. Los resultados fueron referidos a su media, y las diferencias a ésta para cada una de las épocas de observación, se graficaron agrupadas en función de su localización en el mobiliario urbano como guarniciones o banquetas (figura 5), en afloramientos de roca (figura 6) y en obras civiles que sirven como elementos de contención (figura 7).

Como se desprende del análisis de las series de tiempo de las estaciones ubicadas en la zona urbanizada (figura 5) la estación CH55 muestra un desplazamiento en su componente vertical a razón de $4.8 \mathrm{~mm} /$ año desde fines del 2000, pero si se consideran únicamente los 3 últimos años, esta tasa se incrementa a $29 \mathrm{~mm} / \mathrm{año}$. Este resultado es consistente con el hecho de que la estación CH55 es la estación que se ubica más al Occidente dentro de la red geodésica del Chiquihuite y dentro de la zona más baja de piedemonte, por lo que es muy probable que su comportamiento esté afectado por la subsidencia generalizada que se presenta en la zona metropolitana de la Ciudad de México (Cabral et al., 2005). En el resto de los sitios de la red geodésica localizados dentro de la zona urbanizada como son $\mathrm{CH} 15, \mathrm{CH} 25$, $\mathrm{CH} 35$ y CH45, su componente vertical muestra una dispersión ligeramente mayor que las componentes horizontales, lo cual es característico del posicionamiento GPS; sin embargo, estas variaciones tampoco se consideran significativas.

Para las estaciones de la red geodésica del Chiquihuite ubicadas sobre afloramientos de roca $(\mathrm{CH} 10, \mathrm{CH} 30$, $\mathrm{CH} 50$ y CH80), se puede observar (figura 6) que éstas no presentan movimientos significativos y que las variaciones que se muestran se encuentran dentro del límite de error del método, por lo que se concluye que en el lapso estudiado no existe un deslizamiento cuantificable pendiente abajo del talud.

Por otra parte, en las estaciones ubicadas y colocadas sobre elementos de contención en la ladera (figura 7) podemos apreciar que las estaciones $\mathrm{CH} 40, \mathrm{CH} 60 \mathrm{y}$ $\mathrm{CH70}$, aunque muestran una mayor dispersión en sus resultados, tampoco se aprecian en ellas movimientos significativos. Esta situación puede deberse a la geometría de observación de satélites que no resulta ideal, dado que estas estaciones se encuentran cercanas a muros de contención que obstruyen parcialmente la visibilidad hacia el Este, limitando la visibilidad de los receptores GPS hacia algunos satélites y generando una dilución geométrica de la precisión.

El análisis de pendiente y relieve topográfico realizado, indica cuáles pueden ser las zonas de mayor potencial a ser afectadas por deslizamientos del talud y por bloques de roca que se desprenden y ruedan pendiente abajo. Los resultados que se presentan de la red GPS muestran que aunque la colonia La Pastora en la ladera occidental del Cerro Chiquihuite es vulnerable a este tipo de fenómenos, no se han detectado eventos de deslizamiento mayores en la porción alta del talud durante el periodo de observación de octubre del 2000 a diciembre del 2005. Sin embargo, la zona baja del talud, muestra indicios de que está afectada por el proceso de subsidencia en las zonas con mayor contenido de arcillas (Cabral et al., 2005) en el subsuelo. Esta situación indica que las condiciones que provocarían una desestabilización del talud en la ladera Occidental del Cerro del Chiquihuite se mantienen y que probablemente el tiempo de recurrencia para eventos de deslizamiento sea mayor al periodo de observación. 


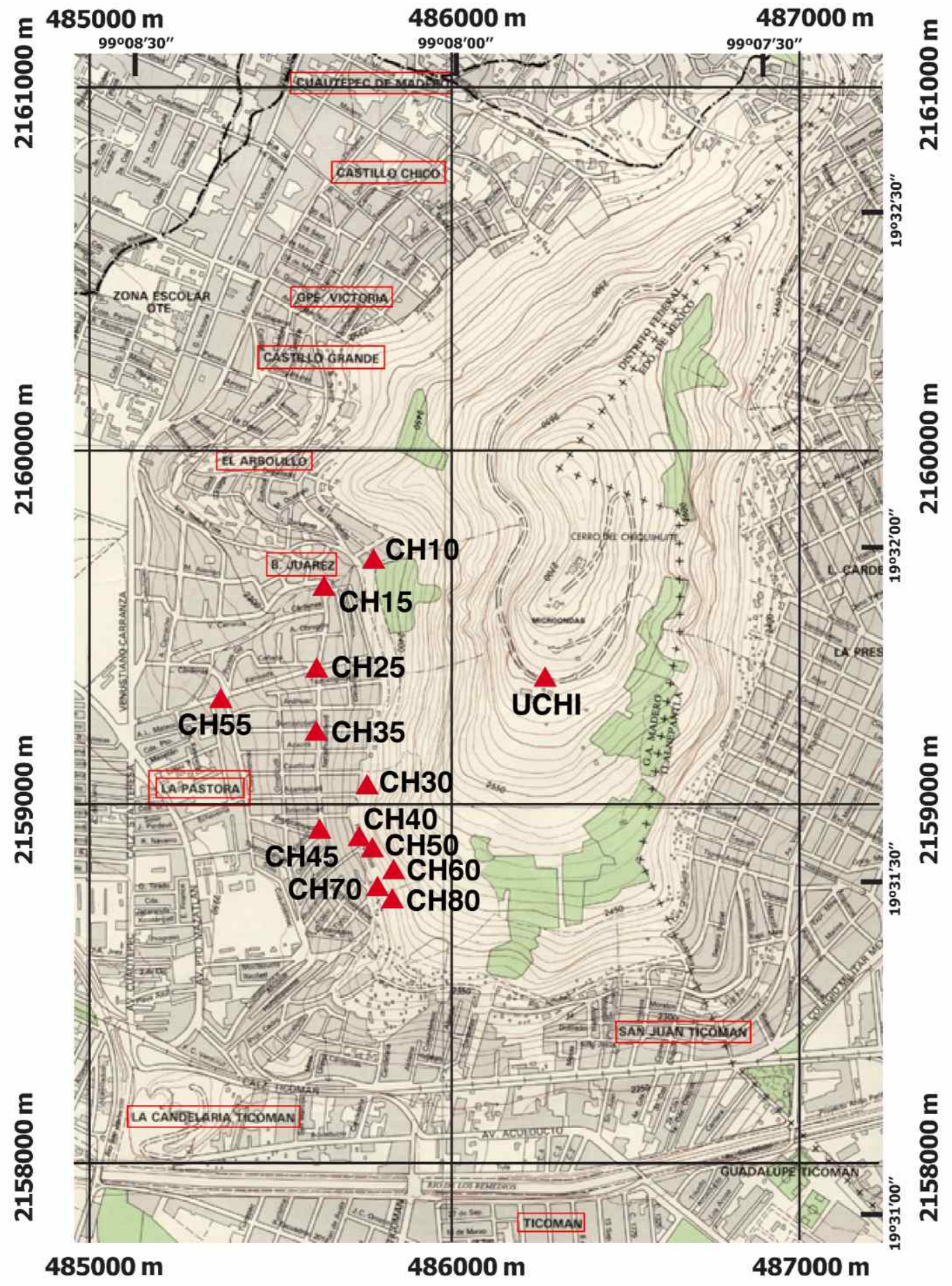

Figura 4. Mapa de localización del Cerro del Chiquihuite. Los triángulos muestran las estaciones GPS utilizadas 
DOI: http://dx.doi.org/10.22201/fi.25940732e.2009.10n2.010

M. Martínez-Yáñez, E. Cabral-Cano, F. Correa-Mora, O. Díaz-Molina, G. Cifuentes-Nava, E. Hernández-Quintero, H. Delgado-Granados

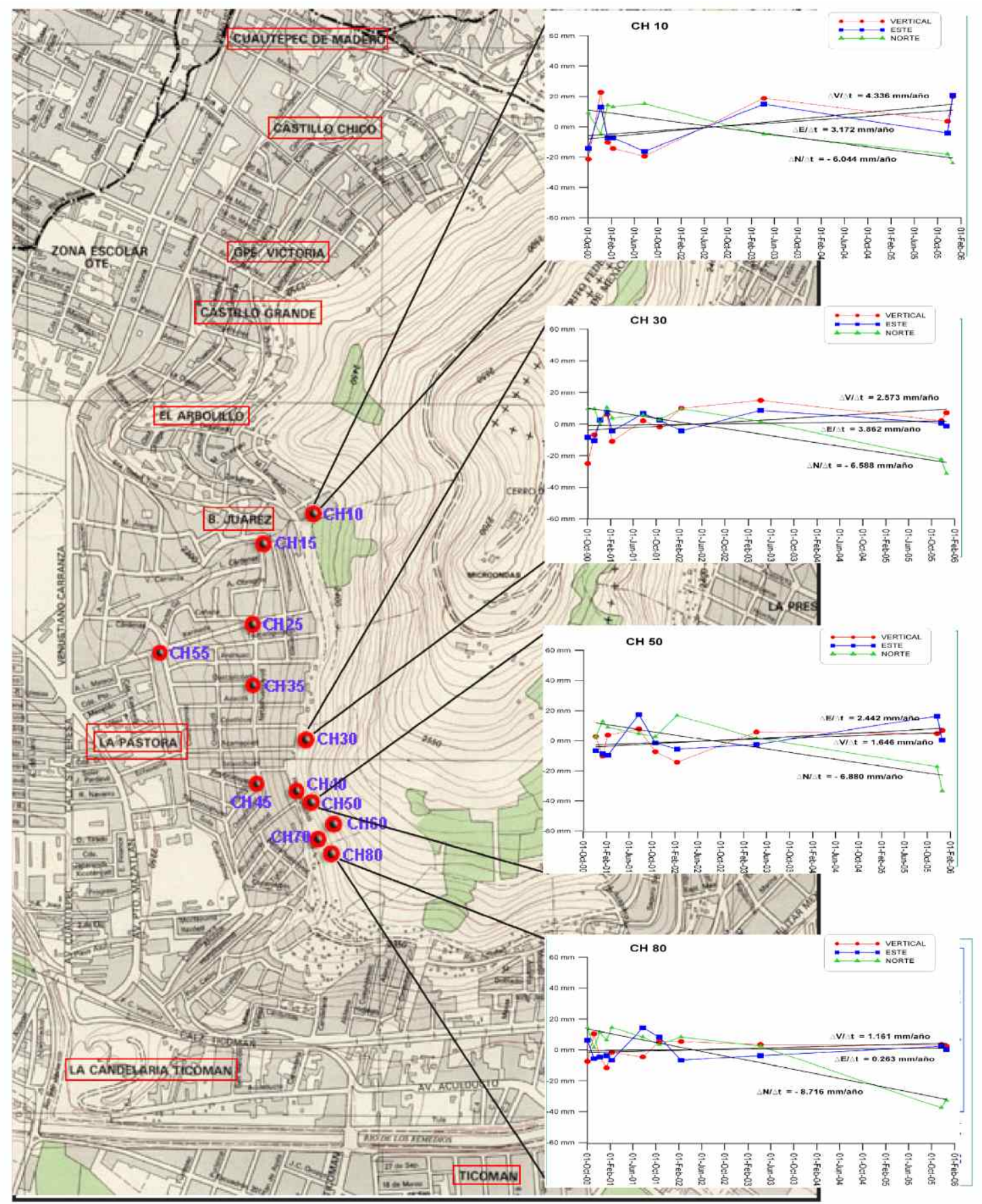

Figura 5. Series de tiempo para las estaciones GPS ubicadas en afloramientos de roca 
DOI: http://dx.doi.org/10.22201/fi.25940732e.2009.10n2.010

Estudio de la ladera occidental del Cerro del Chiquihuite, Ciudad de México mediante SIG y GPS

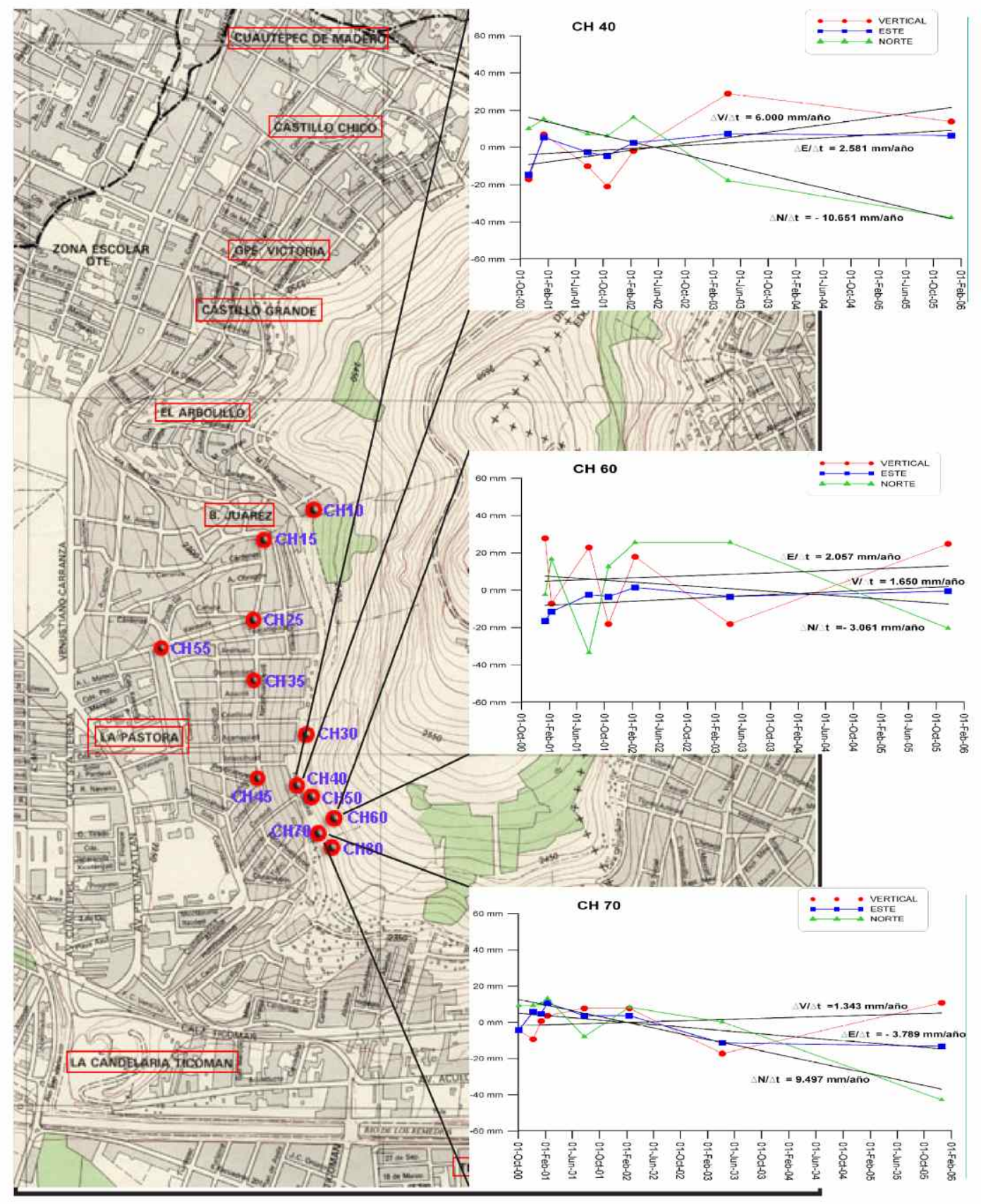

Figura 6. Series de tiempo para las estaciones GPS ubicadas en elementos de contención 
DOI: http://dx.doi.org/10.22201/fi.25940732e.2009.10n2.010

M. Martínez-Yáñez, E. Cabral-Cano, F. Correa-Mora, O. Díaz-Molina, G. Cifuentes-Nava, E. Hernández-Quintero, H. Delgado-Granados

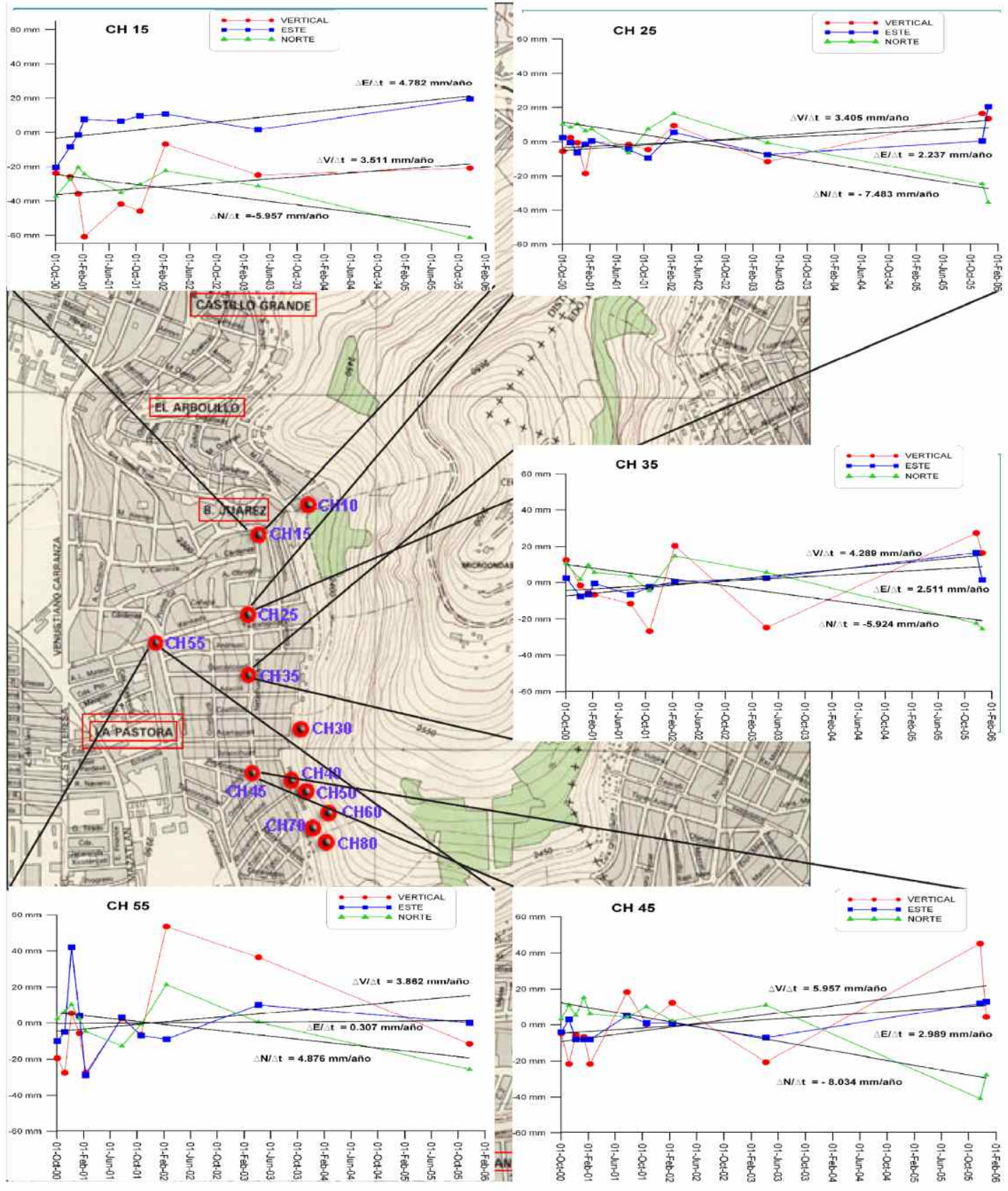

Figura 7. Series de tiempo para las estaciones GPS ubicadas en banquetas 
DOI: http://dx.doi.org/10.22201/fi.25940732e.2009.10n2.010

Estudio de la ladera occidental del Cerro del Chiquihuite, Ciudad de México mediante SIG y GPS

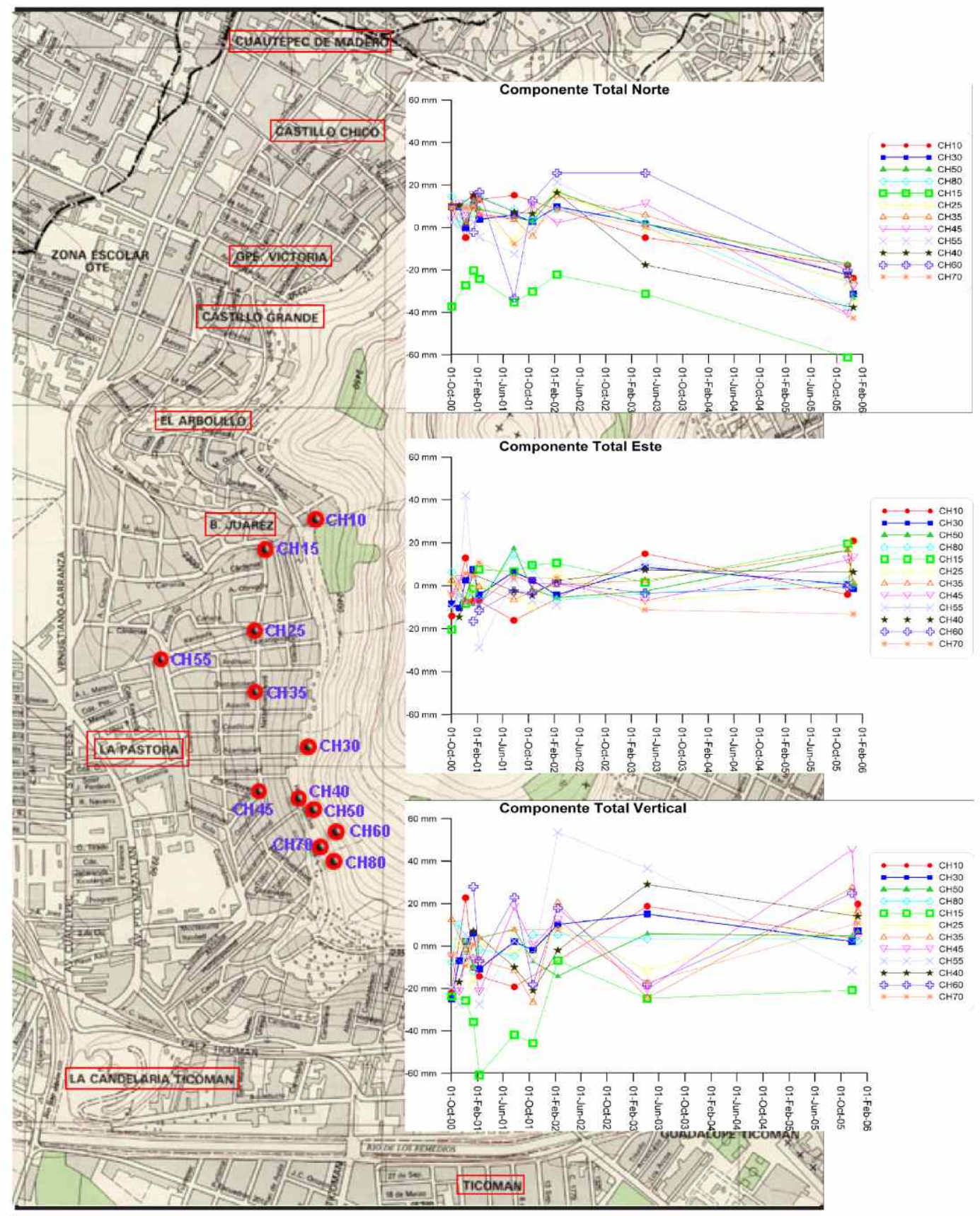

Figura 8. Series de tiempo en componentes Norte, Este y vertical para todas las estaciones GPS ubicadas en el Cerro del Chiquihuite 


\section{Agradecimientos}

Se agradece a las autoridades de la Delegación Gustavo A. Madero las facilidades prestadas para la realización de este trabajo. Asimismo, se agradece al Ejido Cuautepec el uso de sus instalaciones y a la Asociación de Empresas de Telecomunicaciones en el Cerro del Chiquihuite las facilidades prestadas para el acceso y realización de las tareas descritas.

\section{Referencias}

Cabral-Cano E., Dixon T., Díaz-Molina O., Hurtado-Díaz A. Subsidencia en la Ciudad de México, 1984-2005: Intercomparación de resultados mediante técnicas convencionales, Interferometría de Radar de Apertura Sintética y GPS. Reunión Anual Unión Geofísica Mexicana, Puerto Vallarta, Jal., Resúmenes. 2005.
Lugo Hubp J. La disección del relieve en el Sur de la Cuenca de México y porciones adyacentes. Boletín del Instituto de Geografía, (10):55-64. 1981.

Lugo-Hubp J. Mapa geomorfológico del Occidente de la Cuenca de México. Investigaciones Geográficas, (21):9-11. 1990.

Lugo-Hubp J. y Salinas-Montes A. Geomorfología de la Sierra de Guadalupe (al Norte de la Ciudad de México) y su relación con peligros naturales. Revista Mexicana de Ciencias Geológicas, 13(2):240-251. 1996.

Salazar-Mitre L.M. Geología y riesgos asociados en el Valle de México. Riesgos Ambientales para la salud en la Cuenca de México, Programa Universitario del Medio Ambiente, UNAM. México, pp. 267-276. 1996.

Sella G., Dixon T., A. Mao REVEL: A Model for Recent Plate Velocities from Space Geodesy. Jour. Geophys Res., 107(10.1029/2000JB000033). 2002. 
DOI: http://dx.doi.org/10.22201/fi.25940732e.2009.10n2.010

Estudio de la ladera occidental del Cerro del Chiquihuite, Ciudad de México mediante SIG y GPS

\section{Semblanzas de los autores}

Mario Alberto Martínez-Yánez. Cursó la licenciatura de ingeniero topógrafo geodesta en la Facultad de Ingeniería de la UNAM en donde recibió también en el año 2002, la distinción Doctor Gustavo Baz Prada para estudiantes que realizan su servicio social en programas con alto impacto social. Actualmente labora en Dowell Schlumberger de México, SA de CV.

Enrique Cabral-Cano. Es originario de la Ciudad de México, donde cursó la carrera de ingeniería geológica en la Facultad de Ingeniería de la UNAM. Su doctorado lo obtuvo en la Universidad de Miami. Actualmente labora como investigador en el Laboratorio de Cartografía Digital del Instituto de Geofísica de la UNAM y es miembro del Sistema Nacional de Investigadores del Conacyt. Sus intereses académicos incluyen las aplicaciones de sensores remotos, geodesia satelital y exploración geofísica para el análisis de peligros geológicos.

Francisco Correa-Mora. Nacido en la Ciudad de México, es ingeniero geólogo egresado de la Facultad de Ingeniería de la UNAM, con una maestría en geología estructural y tectónica del posgrado en ciencias de la tierra en esta misma universidad. Actualmente está realizando un doctorado en la Universidad de Wisconsin en Madison, EUA. Su campo de investigación está enfocado al modelado de la dinámica de placas tectónicas para la mejor caracterización del riesgo sísmico en México utilizando mediciones de GPS de alta precisión y el método de elemento finito.

Oscar Díaz-Molina. Realizó estudios básicos e intermedios en la población de San Andrés Ahuashuatepec, Tlaxcala. Posteriormente curso la carrera de ingeniero geofísico en la Facultad de Ingeniería, de la UNAM y la maestría en el posgrado en ciencias de la tierra de la UNAM. Desempeña sus labores en el Laboratorio de Cartografía Digital del Instituto de Geofísica. Sus áreas de interés son los sensores remotos, geodesia satelital y la adquisición, procesamiento e interpretación de datos geofísicos para la determinación de zonas de riesgos en zonas urbanas.

Gerardo Cifuentes-Nava. Originario de la Ciudad de México, es ingeniero geofísico por la Facultad de Ingeniería de la UNAM, se desempeña como técnico académico titular en el Instituto de Geofísica, profesor de asignatura de la Facultad de Ingeniería, UNAM y secretario técnico del Instituto de Geofísica UNAM. Sus intereses académicos incluyen geomagnetismo y exploración geofísica. Desarrollo de sistemas para estaciones de registro magnético, instrumentación y mediciones del campo geomagnético. Exploración geofísica somera mediante métodos de tomografía eléctrica, radar de penetración terrestre y métodos potenciales.

Esteban Hernández-Quintero. Es egresado de la Facultad de Ingeniería de la UNAM donde estudió la carrera de Ingeniero Geofísico (1981). Después de trabajar en el Instituto Mexicano del Petróleo, se incorpora al Instituto de Geofísica de la UNAM. Posteriormente obtiene el grado de maestro en ciencias con especialidad en exploración geofísica. Actualmente es jefe del servicio magnético nacional, en la UNAM, además de desarrollar proyectos de geofísica de exploración enfocados al estudio de riesgos naturales.

Hugo Delgado-Granados. Es ingeniero geólogo egresado de la Facultad de Ingeniería de la UNAM, con maestría y doctorado en ciencias por la Universidad Tohoku del Japón. Se desempaña como investigador titular del Instituto de Geofísica de la UNAM, profesor y tutor de la Facultad de Ingeniería y el Posgrado en Ciencias de la Tierra, además de ser miembro del SNI. Su actividad académica comprende evaluación de peligros geológicos, estratigrafía volcánica, relaciones volcanismo-tectónica, geoquímica de gases volcánicos y estudio de glaciares en volcanes activos. 